\title{
RESTOS DE HUEVOS DE DINOSAURIO EN EL CRETÁCICO SUPERIOR DEL SINCLINAL DE VALLCEBRE (BERGUEDÀ, PROVINCIA DE BARCELONA)
}

\author{
Ana María BRAVO ${ }^{1}$, Bernat VILA $A^{2,3}$, Àngel \\ GALOBART ${ }^{3}$ y Oriol $O M S^{4}$
}

${ }^{1}$ Unidad de Paleontología, Dpto. Biología, Universidad Autónoma de Madrid, 28049, Cantoblanco, Madrid. E-mail: ana.bravo@estudiante.uam.es

${ }^{2}$ Consorci Ruta Minera. Carretera de Ribes, 20, 08698, Cercs (Barcelona). E-mail: bernat-vila@mixmail.com

${ }^{3}$ Institut de Paleontologia "M.Crusafont". C/ Escola Industrial, 23, 08201, Sabadell. E-mail: galobartla@diba.es

${ }^{4}$ Universitat Autònoma de Barcelona, Fac. Ciències (Geologia), 08193, Bellaterra (Barcelona). E-mail: joseporiol.oms@uab.es

Bravo, A. M., Vila, B., Galobart, A. \& Oms, O. 2005. Restos de huevos de dinosaurio en el Cretácico Superior del sinclinal de Vallcebre (Berguedà, provincia de Barcelona). [Dinosaur egg remains from the Upper Cretaceous of Vallcebre Syncline (Berguedà, Barcelona Province).] Revista Española de Paleontología, N.E. X, 49-57. ISSN 0213-6937.

\begin{abstract}
Several dinosaur egg remains have been discovered at the materials of the Tremp Formation from the Vallcebre syncline (Upper Cretaceous, south-eastern Pyrenees, Barcelona province). Recent fi eldworks have provided new information about the stratigraphic situation of these remains, accounting for fi fteen new eggsites along three units (Lutitas con Carbones, Lutitas con Oncolitos and Lutitas Rojas inferiores). The abundance of eggs remains along the lithological units suggests an important nesting activity at certain paleoenvironments. Two different oospecies have been identifi ed (Megaloolithus siruguei and Megaloolithus mamillare) which appear at the same or at different stratigraphical levels. The preliminary comparison with the succession of ooespecies described for continental deposits of south of France suggest the presence of one similar association at Vallcebre syncline.
\end{abstract}

KEY WORDS: dinosaurs, eggs remains, oospecies succession, Megaloolithus, Tremp Formation, Upper Cretaceous.

\section{RESUMEN}

Numerosos restos de huevos de dinosaurio han sido hallados en los sedimentos de la Formación Tremp del sinclinal de Vallcebre (Cretácico Superior, Pirineo suroriental, provincia de Barcelona). Los recientes trabajos de campo han proporcionado nueva información acerca de la situación estratigráfi ca de estos restos, conociendo quince nuevos puntos repartidos en tres unidades (Lutitas con Carbones, Lutitas con Oncolitos y Lutitas Rojas inferiores). La abundancia de restos de huevos a lo largo de las unidades litológicas sugiere una importante actividad de nidifi cación en ciertos paleoambientes. Se han identifi cado dos ooespecies distintas (Megaloolithus siruguei y Megaloolithus mamillare) que pueden aparecer en un mismo nivel o bien en diferentes niveles estratigráfi cos. La comparación preliminar con las sucesiones de ooespecies descritas en depósitos continentales del sur de Francia sugiere la existencia de una asociación similar en la sucesión de ooespecies descrita en el sinclinal de Vallcebre.

PALABRAS CLAVE: dinosaurios, restos de huevos, sucesión de ooespecies, Megaloolithus, Formación Tremp, Cretácico superior. 


\section{INTRODUCCIÓN}

El estudio de las cáscaras, huevos y puestas proporciona información sobre la biología reproductiva de los dinosaurios además de información filogenética. Esto es debido a que los huevos son estructuras cuya formación está controlada genéticamente (Kohring, 1997; Grellet-Tinner, 2000). Los hallazgos de cáscaras, huevos y puestas de dinosaurio son relativamente abundantes en la Península Ibérica, especialmente en sedimentos del Cretácico $\mathrm{Su}$ perior (Moratalla, 1993; Sanz et al., 1995; Vianey-Liaud \& López-Martínez, 1997), aunque también hay descritos yacimientos en sedimentos del Cretácico Inferior (Amo, 1998; Amo et al., 2000) y del Jurásico Superior de Portugal (Mateus et al., 1997). Los yacimientos del Cretácico Superior se concentran fundamentalmente en dos áreas: la Cordillera Ibérica y los Pirineos (sectores surcentral y oriental). En la vertiente sur de los Pirineos, las localidades con restos de huevos se sitúan en las zonas de Fontllonga, cuenca de Tremp-Isona, Coll de Nargó y Vallcebre, destacando algunos yacimientos únicos en el registro mundial (Sanz et al., 1995).

El sinclinal de Vallcebre (comarca del Berguedà, provincia de Barcelona; ver figura 1) ha proporcionado numerosos restos de cáscaras de huevo y algunas puestas de dinosaurio a lo largo de la sucesión estratigráfica de la Formación Tremp (Cretácico Superior). Los estudios de este tipo de restos en el sinclinal han sido sin embargo más bien escasos en comparación con otras cuencas del Pirineo surcentral (Sanz et al., 1995; Vianey-Liaud \& López-Martí- nez, 1997; Bravo et al., 2000; López-Martínez et al., 2000). Las primeras citas en la zona de Vallcebre provienen de Aepler (1967) quien identificó cáscaras y huevos de dinosaurios en dicha zona y años más tarde Masriera \& Ullastre (1983) y Médus et al. (1988) citaron también la presencia de cáscaras en la zona del Espà y Vallcebre. Peitz (2000) utilizó cáscaras de la localidad de Gósol para su estudio biométrico y el mismo año se notificó la evidencia más destacada hasta el momento (Galobart et al., 2002). En el presente trabajo se describe el material hallado así como su situación estratigráfica en el sinclinal y se compara de forma preliminar la sucesión estratigráfica de oospecies con evidencias similares del sur de Francia.

\section{MATERIAL Y MÉTODOS}

En el marco de los trabajos de prospección y estudio del Cretácico Superior del sinclinal de Vallcebre se recogieron muestras de cáscaras en distintos niveles estratigráficos del sinclinal y se tomaron datos sobre el diámetro de algunos huevos así como su número y disposición en las puestas. En total se recolectó material de 13 puntos a lo largo del sinclinal a los que se ha asignado una sigla: A-FIG05, D-VAL01, E-MUN02, EMUN03, E-MUN05, I-COM04, I-COM06, I-COM07, I-COM08, N-MAÇ02, N-MAÇ03, N-MAÇ06 y N-MAÇ08. Otras dos localidades (B-FUM10 y E-MUN01) son también consideradas en este estudio a pesar de que no se han analizado en detalle (ver localidades en figura 2 donde cada punto corresponde a un nivel estratigráfico). La posición estratigráfica de algunas de ellas (D-VAL01, I-COM06, I-COM07 e I-COM08), podría admitir
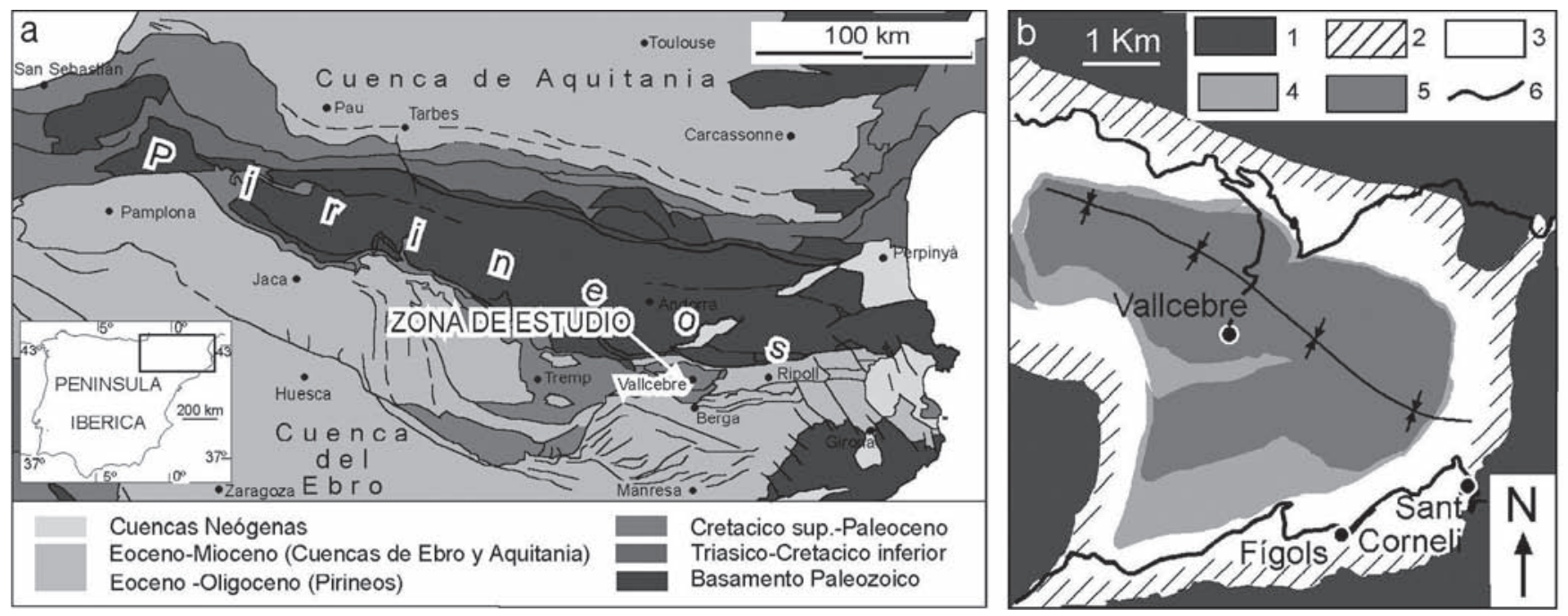

Figura 1. a: Mapa geológico general de los Pirineos con la situación de la zona de estudio. b: Esquema geológico del sinclinal de Vallcebre (1: Cretácico marino, 2: "Garumniense" Gris, 3: "Garumniense” Rojo inferior, 4: Calizas de Vallcebre, 5: "Garumniense" suprayacente a las Calizas de Vallcebre y 6: carreteras.)

a: Geological sketch map of the Pyrenees with location of the studied area. b: Geological sketch of the Vallcebre syncline (1: marine Cretaceous, 2: Grey "Garumnian", 3: lower Red "Garumnian", 4: Vallcebre limestones, 5: "Garumnian" overlain the Vallcebre limestones and 6: roads). 
una oscilación de unos 20 metros en la vertical. Este hecho se debe a las condiciones locales tectónicas y de afloramiento que dificultan una correlación precisa. Dada la distribución vertical de los yacimientos en la sección, esta oscilación no supone ninguna alteración de la sucesión de localidades.

Tras eliminar los depósitos secundarios por medio de un baño de ultrasonidos se estudiaron las superficies interna y externa de las cáscaras mediante una lupa binocular. Los huevos de la puesta del yacimiento E-MUN05 fueron sometidos a distintos tratamientos químicos (ácido acético al 15\%, hexametafosfato sódico al 10\%, agua, carbonato de amonio al $15 \%$ e hidróxido potásico), así como a tomografias computerizadas (TAC) para la evaluación de su posible contenido embriológico. Para el estudio de la forma y proporciones de las unidades cristalinas, así como del sistema de canales, se hicieron láminas delgadas en vista radial de algunas muestras mientras que otras fueron montadas en soportes de aluminio y cubiertas con un baño de oro para su estudio con el microscopio electrónico de barrido. La mineralogía de algunas muestras fue analizada mediante patrones de difracción de rayos X (EDAX).

\section{CONTEXTO GEOLÓGICO}

Los restos de huevos de dinosaurio se localizan en los materiales continentales y de transición de la Formación Tremp en el sinclinal de Vallcebre (unidad manto inferior del Pedraforca, Pirineos sur-orientales). La sucesión cretácica de las clásicamente conocidas como facies "Garumniense" presenta en el sinclinal de Vallcebre una potencia total de cerca de 500 metros (Fig. 2) y ha sido dividida en seis unidades según criterios litológicos y sedimentológicos (Aepler, 1967; Oms et al., 2002). Los huevos y cáscaras se conservan en las unidades Lutitas con Carbones, Lutitas con Oncolitos y Lutitas Rojas inferiores. Las dos primeras unidades corresponden al "Garumniense" Gris y la tercera corresponde a la unidad inferior del "Garumniense" Rojo de la clasificación regional de Rosell et al. (2001) (Fig. 2), registrando el tránsito de ambientes de transición de facies supramareales y lacustres a ambientes fluviales.

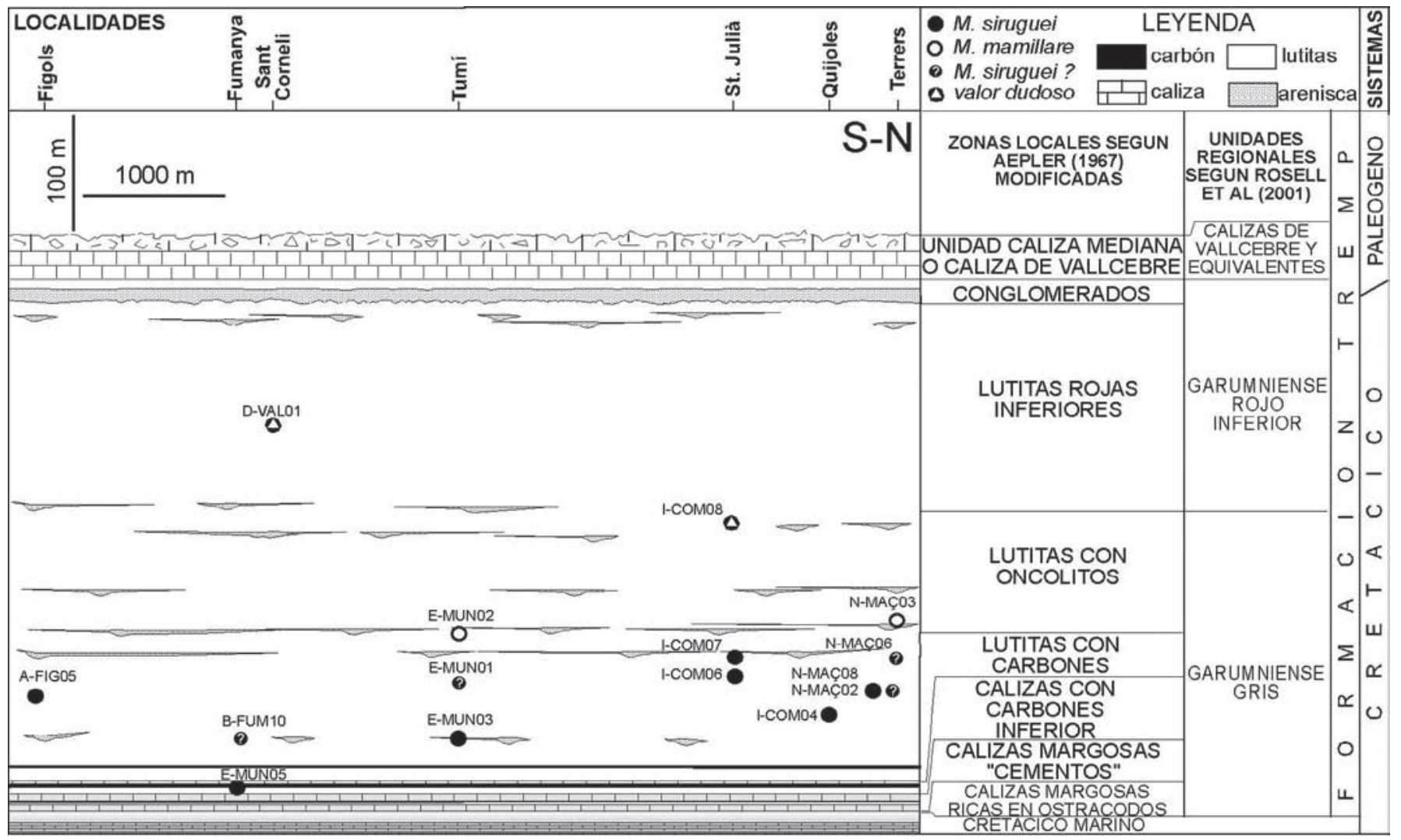

Figura 2. Situación estratigráfica de las localidades con restos de huevos en el sinclinal de Vallcebre (con datos de Oms et al., 2002). Los yacimientos B-FUM10 y E-MUN01 no han sido analizados en detalle aunque podrían representar evidencias de $M e$ galoolithus siruguei Vianey-Liaud et al., 1994.

Stratigraphic setting of the eggsites localities at Vallcebre syncline (with data from Oms et al., 2002). The eggsites BFUM10 y E-MUN01 have not been analyzed in detail but could represent evidences of Megaloolithus siruguei VianeyLiaud et al., 1994. 
La sucesión vertical de las facies "Garumniense" cretácicas indica una evolución desde condiciones transicionales y claramente reductoras hasta condiciones oxidantes. A su vez, se observa cómo los fósiles de huevos son más frecuentes en las facies reductoras.

La unidad "Lutitas con carbones" está formada por más de 100 metros de limos y arcillas de tonos grises y oscuros con intercalaciones decimétricas de capas de lignitos, areniscas de grano fino o muy fino y calizas con carófitas. $\mathrm{Su}$ contenido fósil está formado principalmente por gasterópodos diversos (ej. niveles con Lychnus, cerítidos y potamídidos) y bivalvos (ej. Corbicula, ostréidos) de aguas salobres, ostrácodos y carófitas (Feist \& Colombo, 1983), tapices algales, huevos y fragmentos de cáscaras de huevo de dinosaurio y otros restos óseos de vertebrados. En general, se observa un marcado proceso de bioturbación por raíces y concentración de nódulos de sulfuros que, por lo menos en parte, parecen estar asociados a procesos de bioturbación. Todo esto permite inferir unas condiciones dominantemente reductoras.

La unidad "Lutitas con oncolitos" está formada por cerca de 100 metros de lutitas ocres y violáceas con un marcado proceso de burrowing vertical, areniscas de grano fino e intercalaciones menores de calizas lacustres y niveles con oncolitos (Aepler, 1967; Oms et al., 2002). El contenido fósil de la unidad está formado principalmente por gasterópodos y bivalvos dulceacuícolas (ej. uniónidos), cáscaras y huevos de dinosaurio y restos óseos de vertebrados. Este segundo conjunto de facies lo interpretamos como fluvio-lacustre con amplias zonas de inundación donde también sería frecuente el desarrollo de paleosuelos.

La unidad "Lutitas rojas inferiores" está formada por cerca de 150 metros de lutitas rojas, niveles de areniscas y conglomerados (restringidos a la zona oeste del sinclinal). Su contenido fósil está formado por carófitas, ostrácodos, palinomorfos (Feist \& Colombo, 1983; Médus et al., 1988), huesos, huellas y cáscaras de huevos de dinosaurio. Se interpreta como facies de ambientes fluviales con unas condiciones claramente oxidantes.

Tanto los fragmentos de cáscaras como los huevos enteros se encuentran en dos tipos de facies comunes a las tres unidades mencionadas. Una primera facies está formada por capas canaliformes de arenisca fina o muy fina con claras superficies de acreción lateral que se formaron en un ambiente confinado. Una segunda facies está formada por capas tabulares de limos o pelitas que se interpretan como sedimentos desconfinados). Estas facies desconfinadas se habrían depositado en una llanura de inundación dominada por procesos de baja energía pero que genéticamente estarían relacionados con fácies de canales. Según Rosell et al. (2001) y Aepler (1967) estas facies corresponderían a ambientes transicionales en el "Garumniense" gris y fluviales en el "Garumniense" rojo inferior. La adscripción de estas facies (en particular las que presentan un carácter relativamente reductor) a un ambiente mareal (que seria

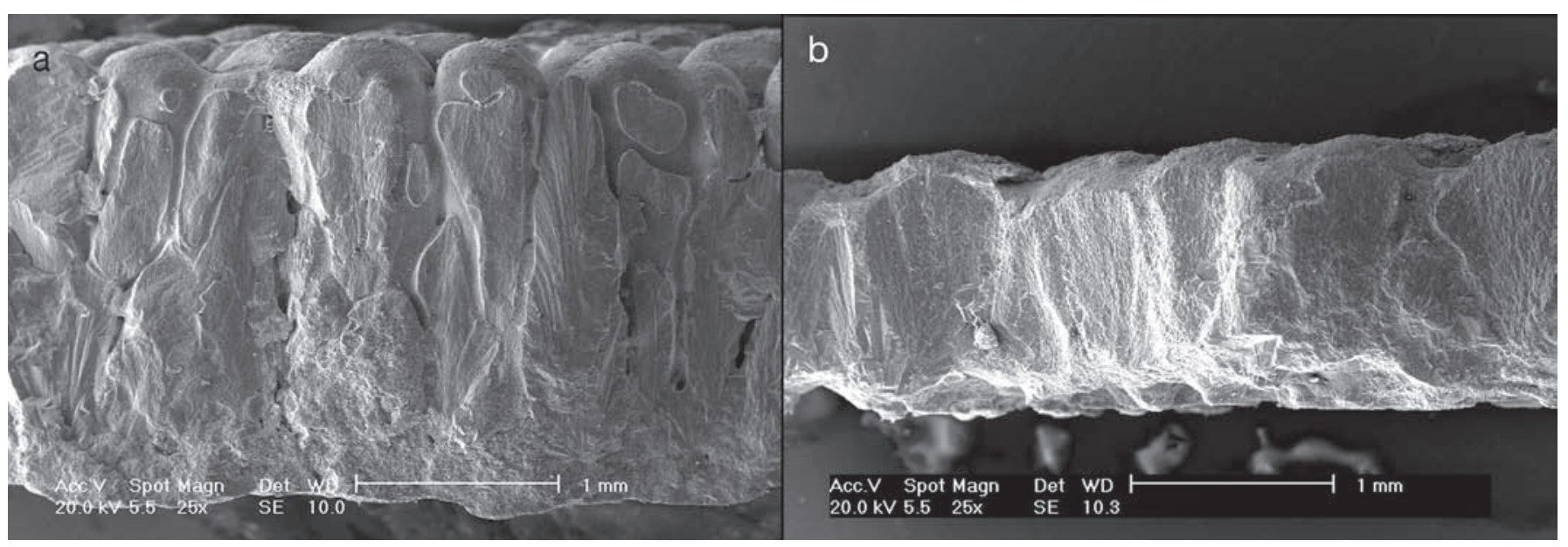

Figura 3. Fotografías al microscopio electrónico de barrido de dos fragmentos de cáscara en vista radial. a: Fragmento perteneciente a la ooespecie Megaloolithus siruguei Vianey-Liaud et al., 1994 (muestra E-MUN03). Las unidades cristalinas son alargadas y entre ellas se pueden ver algunos canales respiratorios rectos unidos por canales transversales. b: Megaloolithus mamillare Vianey-Liaud et al., 1994 (muestra N-MAÇ03). En este caso, las unidades cristalinas son más anchas que altas y los canales respiratorios son rectos u oblicuos pero sin canales transversales.

Scanning electron photographs of two eggshell fragments in radial view. a: Fragment belonging to the oospecies Megaloolithus siruguei Vianey-Liaud et al., 1994 (sample: E-MUNO3). The eggshell units are elongated and between them run the respiratory channels more or less straight and connected by transversal ones. $\boldsymbol{b}$ : Megaloolithus mamillare Vianey-Liaud et al., 1994 (sample N-MAÇ03). In this case the crystalline units are more wide than long and the respiratory channels are straight or oblique but without transversal channels. 
verosímil en un contexto general de evolución vertical de un ambiente marino hacia continental) no se considera puesto que en ningún caso se han encontrado características de tales ambientes (presencia de ciclos de neap-spring, bidireccionalidad mareal del flujo, etc).

\section{DESCRIPCIÓN DEL MATERIAL}

Los fragmentos de cáscara y puestas de dinosaurio hallados en el sinclinal de Vallcebre pertenecen a la oofamilia Megaloolithidae Zhao, 1979. Esta oofamilia se encuentra ampliamente distribuida en el noroeste de la Península Ibérica, sur de Francia y La India (Vianey-Liaud et al., 1994; Sahni et al., 1994) aunque también se han citado yacimientos en Rumanía y Argentina (Grigorescu et al., 1994; Chiappe et al., 1998).

Del conjunto de evidencias de huevos y cáscaras se han distinguido fundamentalmente tres tipos de localidades atendiendo al grado de preservación: 1) localidades con huevos enteros, fragmentos de cáscaras asociados y/o evidencia de puesta con diversos huevos (A-FIG05, B-FUM10, E-MUN01, E-MUN03, E-MUN05, I-COM04, I-COM06, I-COM07, N-MAÇ06 у N-MAÇ08); 2) fragmentos de cáscara (D-VAL01, E-MUN02, I-COM08, N-MAÇ02 y N-MAÇ03), y 3) fragmentos de cáscara incorporados como clastos en cuerpos sedimentarios tractivos (muestras no consideradas en este trabajo). En el primer caso se ha podido distinguir el contorno de los huevos in situ en diferentes estados de conservación y en una ocasión se ha podido recuperar todo el conjunto de huevos (localidad E-MUN05). En otras se han distinguido, en un mismo nivel estratigráfico, distintas zonas con puestas, huevos completos y cáscaras asociadas (localidad A-FIG05, con posibles efectos de expolio). El mismo tipo incluye los fragmentos de cáscaras que aparecen en lutitas en acumulaciones más o menos abundantes. Esta concentración y disposición de cáscaras sugiere la presencia de antiguas puestas por lo que podría ser interesante una excavación (ej. E-MUN01, A-FIG05). El segundo tipo corresponde a los fragmentos de cáscaras asociados a lutitas (ej. E-MUN02) dónde, hasta el momento, no ha sido posible identificar huevos enteros o concentraciones significativas, aunque su hallazgo parece más probable que en el tipo 3 . El tercer caso difiere del anterior en que los fragmentos de cáscara aparecen totalmente aislados, sin formar una acumulación, dentro de cuerpos areniscosos evidenciando un transporte de éstos como partículas detríticas y, en consecuencia, con pocas posibilidades de hallar huevos o puestas enteras.

El material estudiado se encuentra depositado en el Institut de Paleontología M. Crusafont de Sabadell (siglas IPS 27361, 27362, 27363, 27364, 27365, 27366, 27367, 27378, 27380, $27381,27382,27383,27388,27376,27379,27385,27386$, 27387 y 27853$)$.

\section{-Puestas}

En el conjunto de localidades del sinclinal se han distinguido una decena de puntos con concentraciones de huevos que representan o podrían representar puestas. Los puntos descritos corresponden a localidades del tipo tafonómico 1 y presentan una variabilidad de 2 a 5 huevos por puesta. La gran mayoría de ellas están formadas por huevos de la ooespecie Megalooli- thus siruguei (A-FIG05, E-MUN05, I-COM04, I-COM06, ICOM07, N-MAÇ06 y N-MAÇ08) y hasta el momento no se han hallado huevos de la ooespecie Megaloolithus mamillare. Algunas de las puestas de la localidad A-FIG05 no han podido ser asignadas a una ooespecie aunque si se pueden identificar como pertenecientes al oogénero Megaloolithus. Futuros análisis de nuevo material permitirán la identificación dichas puestas. Existen otras localidades (B-FUM10, E-MUN01, E-MUN03) en las cuales es necesaria una excavación para comprobar la presencia inequívoca de puestas en puntos con acumulaciones de cáscaras o huevos aislados.

Entre las puestas halladas destacan las descubiertas durante las tareas de actividad minera del carbón en la zona sur del sinclinal (muestra E-MUN05; Fig.4) (Galobart et al., 2002). Las puestas en esta localidad perteneciente a la unidad Lutitas con Carbones, están situadas dentro y probablemente también a techo de una arenisca fluvial de grano muy fino, que muestra varias unidades de acreción resultado de la migración del canal. En el cuerpo areniscoso se han diferenciado dos puestas consecutivas separadas por un nivel de acreción de varios decímetros de potencia. Lateralmente se observan, en una nueva acreción, huevos que podrían indicar una tercera puesta. Algunos huevos permanecen aún in situ mientras otros fueron extraídos y restaurados. La mayoría de los huevos están prácticamente completos y presentan una forma subesferica ligeramente comprimida por uno de sus ejes y un diámetro aproximado de unos $15-20 \mathrm{~cm}$ (Fig. 4). El sedimento que rellena éstos es homogéneo y de grano fino, igual que la matriz en que los huevos están envueltos, como corroboró la exploración empleando el Escáner Tomográfico Computerizado. Aunque se recuperaron casi la totalidad de los huevos en su agrupación original no se pudo determinar una morfología detallada del nido, ni establecer con certeza a cual de las dos acumulaciones podían pertenecer. Tanto los aspectos tafonómicos como los sedimentológicos descartan una acumulación tractiva de los huevos y sugieren una actividad de nidificación recurrente en las distintivas etapas de acreción de la arenisca fluvial. Cabe resaltar la presencia inequívoca de huevos dentro de las facies de relleno de canal (areniscas muy finas). Este hecho indica una nidificación en sedimentos de texturas sensiblemente variadas (tanto lutítica como limosaareniscosa muy fina).

El resto de puestas (puntos A-FIG05, I-COM04, I-COM06, I-COM07, N-MAÇ06 y N-MAÇ08) presentan un tamaño más reducido conteniendo sólo 2 huevos en las más pequeñas y de 3 a 4 (incluso 5 en algún caso), en las más numerosas. Estos son de contorno circular y aparecen en sección rellenos del mismo sedimento que los rodea. Excavaciones futuras en las localidades permitirán comprobar o ampliar estos datos preliminares.

\section{-Cáscaras}

El estudio del material procedente del sinclinal de Vallcebre ha permitido identificar dos oospecies. Los restos analizados corresponden a localidades del tipo tafonómico 1 y 2, tanto en puntos con presencia de puestas como en acumulaciones de cáscaras. La mayoría de los puntos de un mismo nivel estratigráfico contiene una única ooespecie, aunque en algún caso será necesario analizar nuevo material para su identificación definitiva (localidad A-FIG05). 


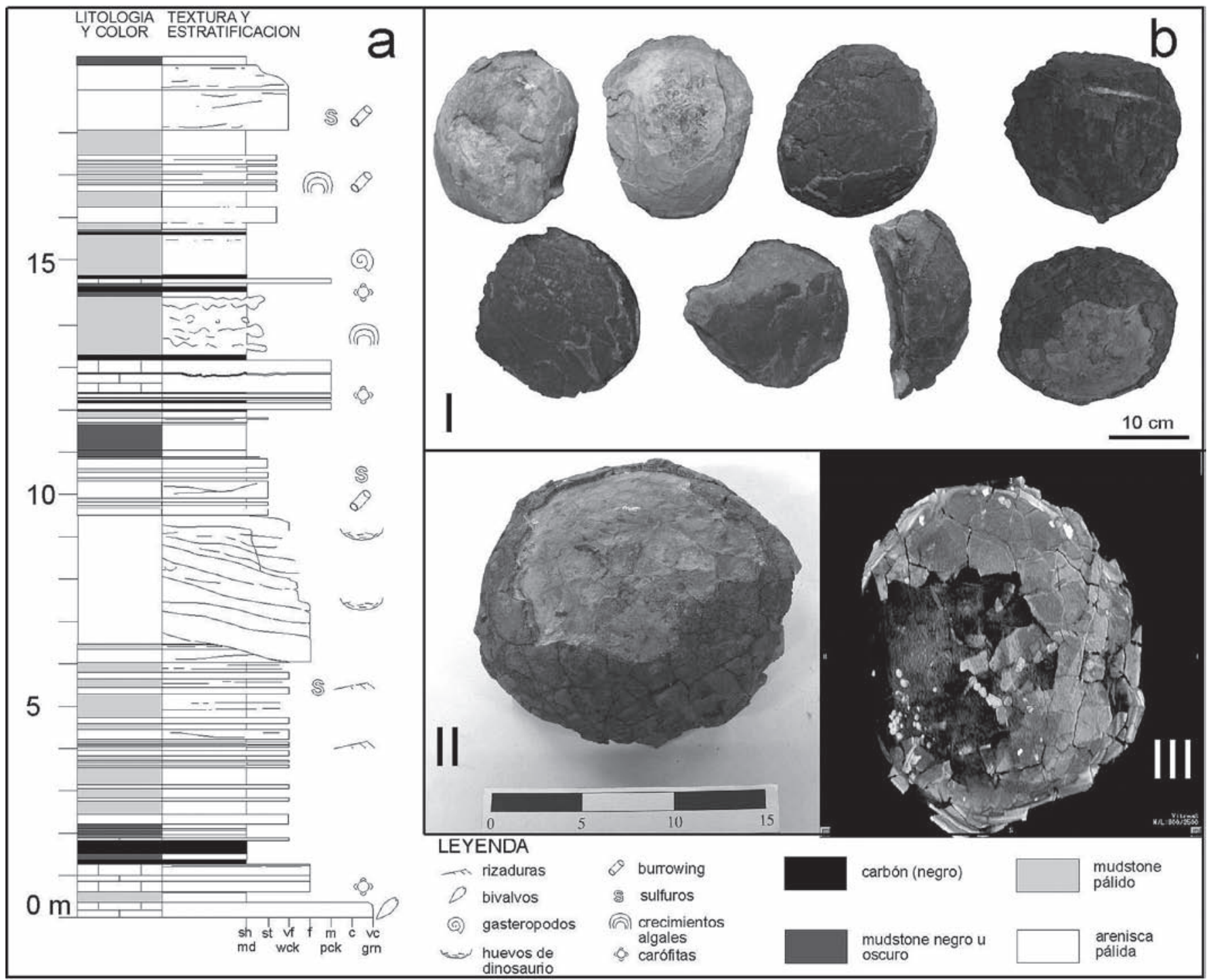

Figura 4. a: Sección estratigráfica de las puestas de la localidad E-MUN05 en el sinclinal de Vallcebre. b: Megaloolithus siruguei Vianey-Liaud et al., 1994 de la puesta de E-MUN05. I- conjunto de huevos recuperados; II- un huevo de la puesta; IIIreconstrucción del huevo en 3D.

a: Stratigraphic section from E-MUN05 eggsite at Vallcebre syncline. $\boldsymbol{b}$ : Megaloolithus siruguei Vianey-Liaud et al., 1994 from the clutch at E-MUN05 eggsite. I- group of recovered eggs; II- an egg from the clutch; III- 3D reconstruction of the egg.

Ooespecie: Megaloolithus siruguei Vianey-Liaud et al. 1994

Figs. 3a y 4

Muestras: parte del material de A-FIG05, E-MUN03, EMUN05, I-COM04, I-COM06, I-COM07, I-COM08, N-MAÇ02, N-MAÇ06, N-MAÇ08 y D-VAL01, y probablemente B-FUM10 y E-MUN01.

Descripción: Superficie externa cubierta por nodos compactados, de aspecto redondeado o ligeramente poligonal (diámetro de los nodos entre 0,3-1,1 mm). Los poros se disponen alrede- dor de los nodos en los intersticios existentes entre ellos. Las unidades cristalinas son de tipo esferulítico, alargadas, más altas que anchas (índice altura (AT) /anchura (AN) de las unidades entre 2,60-3,30) y de bordes laterales casi paralelos. Las líneas de crecimiento aparecen curvadas con la convexidad hacia el exterior desde la base al extremo de las unidades. Los canales respiratorios se sitúan entre las unidades cristalinas, son relativamente rectos y se encuentran unidos por otros canales transversales formando una red tridimensional compleja (Fig. 3a). La superficie interna está cubierta por mamilas de contorno circular. El análisis por medio de la sonda EDAX muestra que las cáscaras están compuestas de carbonato cálcico. Grosor de la cáscara entre $1,93-3,27 \mathrm{~mm}$. 


\section{Ooespecie: Megaloolithus mamillare Vianey- Liaud et al., 1994}

Fig. $3 b$

\section{Muestras: E-MUN02, N-MAÇ03.}

Descripción: Superficie externa cubierta por nodos redondeados (diámetro entre $0,4-1 \mathrm{~mm}$ ) a menudo fusionados y algunos de un tamaño mayor que los otros. Unidades esferulíticas en forma de abanico, más anchas que largas (Fig. 3b) (índice AT/AN: $2,10-2,60$ ) con líneas de crecimiento ligeramente curvadas con la convexidad hacia el exterior desde la base hasta el extremo de las unidades y que en algunos casos continúan entre unidades. Sistema de canales de tipo tubocanaliculado (Mikhailov, 1997) aunque hay algunos canales oblicuos que se fusionan y abren al exterior en un único poro. El análisis por medio de la sonda EDAX muestra que las cáscaras están compuestas de carbonato cálcico. Grosor de la cáscara: 1,64-2 mm.

\section{DISCUSIÓN}

Las gran mayoría de las cáscaras del yacimiento de Vallcebre pertenecen a la ooespecie Megaloolithus siruguei Vianey-Liaud et al., 1994. Esta ooespecie se caracteriza fundamentalmente por presentar unas unidades cristalinas alargadas y un sistema de canales complejo formado por una red tridimensional de canales. La segunda ooespecie hallada en el sinclinal de Vallcebre es Megaloolithus mamillare Vianey-Liaud et al., 1994 que se diferencia claramente de $M$. siruguei en la forma y proporción de las unidades (en forma de abanico y más anchas que altas) y en el sistema de canales (con canales rectos u oblicuos pero nunca con canales transversales). Aunque el diámetro de los nodos es muy similar, en este caso los de M. mamillare son de aspecto más redondeado y ligeramente más grandes que los de M. siruguei. Otra diferencia radica en la fusión de nodos, que es relativamente frecuente en $M$. mamillare mientras que en $M$ .siruguei ocurre muy raramente.

Algunas de las puestas halladas en la localidad A-FIG05 no han podido ser atribuidas claramente a ninguna ooespecie. Las cáscaras pueden atribuirse claramente al oogénero Megaloolithus, pero es necesario el análisis de nuevo material para poder identificar a qué ooespecie pertenecen.

Las puestas del yacimiento de Vallcebre pertenecientes a la ooespecie Megaloolithus siruguei contienen en su mayoría entre 2 y 3 huevos (a veces hasta 4), mientras que las halladas en el sur de Francia pueden contener entre 3 y 5 huevos, (Garcia, 1998). En el caso de la ooespecie Megaloolithus mamillare, se han descrito puestas con 4 huevos (Garcia, 1998) mientras que no se han identificado hasta el momento puestas de esta ooespecie en Vallcebre. El número de huevos por puesta varía, siendo las del sinclinal de Vallcebre ligeramente más pequeñas que las descritas en yacimientos del sur de Francia y en otros yacimientos de la Península ibérica como Biscarri (López-Martínez et al., 2000) y Faidella (Bravo et al., 2000). No obstante, en algunos casos el enterramiento de los huevos a distintas profundidades es responsable de que tras la posterior erosión no se pueda estimar el número exacto de huevos por puesta y sólo su forma exacta en los más superficiales, a menudo parcialmente conservados. Por lo tanto se trata de datos no definitivos que habrá que ampliar en futuras excavaciones.

Las dos ooespecies halladas en los sedimentos del sinclinal de Vallcebre aparecen en niveles sucesivos. Tres asociaciones distintas de oospecies pertenecientes a la oofamilia Megaloolithidae han sido descritas en sedimentos continentales del sur de Francia (Garcia \& Vianey-Liaud, 2001). Una de ellas es la formada por las ooespecies Megaloolithus siruguei, M. petralta, M. aureliensis, M.microtuberculata, Cairanoolithus dughii y C. roussentensis que caracterizaría el Campaniense superior, una segunda que incluye a $M$. siruguei, $M$. petralta y M. mamillare (finales del Campaniense comienzo del Maastrichtiense) y por último la asociación de $M$. mamillare y M. pseudomamillare (Maastrichtiense). En el sinclinal de Vallcebre encontramos una asociación formada básicamente por $M$. siruguei y $M$. mamillare, similar a la segunda descrita en el sur de Francia, aunque con algunas diferencias. Así por ejemplo, aunque $M$. siruguei aparece de nuevo a continuación de $M$. mamillare (Fig.2) en las localidades donde esto ocurre (I-COM08 y D-VAL01) se han hallado únicamente fragmentos de cáscara lo que sugiere que pueden haber sido transportados y por lo tanto su valor tafonómico y cronoestratigráfico es dudoso. Por otro lado, la correspondencia en edades de la asociación identificada en el sinclinal de Vallcebre con las asociaciones francesas no está por el momento justificada y queda a la espera de obtener nuevas calibraciones paleomagnéticas. Un análisis detallado de la estructura y preservación de las cáscaras así como nuevas prospecciones y dataciones son necesarias para ampliar el conocimiento de esta asociación preliminar.

La disminución en abundancia de zonas con puestas y cáscaras hacia unidades más superiores en la evolución vertical de las facies "garumniense" cretácicas de la Formación Tremp sugiere un posible sesgo tafonómico, en el sentido de que el registro sería discontinuo y probablemente menos diverso hacia el techo de la sucesión. Ello podría estar estrechamente ligado a factores ambientales. En efecto, en la parte superior cada vez abundan facies más oxidantes que podrían dificultar la conservación de los huevos o podría representar la existencia de ambientes paleogeográficos poco idóneos para la puesta. Por el contrario, las facies reductoras acumulan la gran mayoría de localidades. 


\section{CONCLUSIONES}

El estudio preliminar de los fragmentos de cáscaras del sinclinal de Vallcebre muestra la presencia de dos ooespecies que aparecen sucesivamente en niveles distintos. Por lo tanto se establece claramente una sucesión de ooespecies a lo largo de los niveles cretácicos de la Formación Tremp en el sinclinal y sería también plausible proponer una asociación preliminar (M. siruguei seguido de $M$. mamillare) a lo largo de las distintas unidades, de forma parecida a lo descrito en algunas localidades de edad similar del sur de Francia aunque con ciertas reservas. Las características de las puestas en la ooespecie $M$. siruguei de Vallcebre sugieren también una posible similitud con otras descritas en Francia aunque sería necesario realizar excavaciones detalladas de las puestas con el fin de analizar su estructura y testificar un probable sesgo preservacional. La abundancia de la ooespecie $M$. siruguei en la unidad "Lutitas con carbones" y su disminución hacia unidades más superiores donde predomina aunque en menor grado la ooespecie $M$. mamillare, podría estar relacionado con cambios en las condiciones paleoambientales de los lugares de puesta, con un sesgo positivo hacia facies más reductoras.

\section{AGRADECIMIENTOS}

Este estudio se enmarca en el "Projecte de recerca dels jaciments paleontològics del trànsit Mesozoic-Cenozoic continental català: implicacions paleogeogràfiques i biogeogràfiques" (Servei de Patrimoni del Departament de Cultura de la Generalitat de Catalunya) y sus actividades de intervención. Agradecemos al Consorci Ruta Minera la colaboración y apoyo en la realización del estudio. Este estudio ha sido posible gracias al programa INTERREG IIIA. El análisis de las muestras ha sido posible gracias a un proyecto de la Jurassic Foundation concedido a uno de los autores (A.M. Bravo). Queremos agradecer a la Dra. López-Martínez y al Dr. Moratalla sus sugerencias y comentarios que han contribuido a la mejora del trabajo. Agradecemos también a R. Gaete, A. Buscalioni, S. Val, G. Muller e I. Pellegero su colaboración en los trabajos preliminares y a J. Llobet por la notificación de una de las localidades.

\section{BIBLIOGRAFÍA}

Aepler, R. 1967. Das garumnian der Mulde von Vallcebre und ihre Tektonik (Spanien, Provinz Barcelona). Master Thesis Freien Universität Berlin (Naturwissenschaftlichen Fakultät), 101 pp. (inédito).

Amo, O. 1998. Fragmentos de cáscara de huevo de vertebrados del Cretácico inferior de Galve (Teruel). Tesis de Licenciatura. Universidad de Zaragoza, 116 pp. (inédita).

Amo, O., Canudo, J.I. \& Bescos, G. 2000. First record of Elongatoolithid eggshells from the lower Barremian
(Lower Cretaceous) of Europe (Cuesta Corrales 2, Galve, Teruel, Spain)". First International Symposium on Dinosaur Eggs and Babies, Isona, Extended Abstracts, 7-13.

Bravo, A.M., Moratalla, J.J., Santafé. J.V. \& Santisteban, C. 2000. Faidella, a new Upper Cretaceous Nesting Site from the Tremp Basin (Lérida Province, Spain). First International Symposium on Dinosaur Eggs and Babies, Isona. Extended Abstracts, 15-21.

Chiappe, L. M., Coria, R. A., Dingus, L., Jackson, F., Chinsamy, A. \& Fox, M. 1998. Sauropod dinosaur embryos from the Late Cretaceous of Patagonia. Nature, 396, 258-261.

Feist, M. \& Colombo, F. 1983. La limite Cretacé-Tertiaire dans le nord-est de l'Espagne du point de vue des carophytes. Geologie Méditerranée, 10, 303-326.

Galobart, À., Oms, O., Gaete, R., Buscalioni, A., Bravo, A.M., Val, S., Muller, G., \& Pellegero, I. 2002. Preliminary study of a dinosaur eggs nest from the Maastrichtian of Fumanya (Catalan Pyrenees). I Congreso Internacional sobre Dinosaurios y otros Reptiles Mesozoicos en España. Resúmenes.

Garcia, G. 1998. Les coquilles d'oeufs de dinosaures de Crétacé supérieur de Sud de la France. Diversité, paléobiologie, biochronologie et paléoenvironnements. Tesis Doctoral. Université de Montpellier II, France, 152 pp. (inédita).

Garcia, G. \& Vianey-Liaud, M. 2001. Dinosaur eggshells as biochronological markers in Upper Cretaceous continental deposits. Palaeogeography, Palaeoclimatology, Palaeoecology, 169, 153-164.

Grellet-Tinner, G. 2000. Phylogenetic interpretation of eggs and eggshells of Paleognathae. First International Symposium on Dinosaur Eggs and Babies, Isona. Extended Abstracts, 61-75.

Grigorescu, D., Weishampel, D., Norman, D., Seclamen, M., Rusu, M., Baltres, A. \& Teodorescu, V. 1994. Late Maastrichtian dinosaur eggs from the Hateg Basin (Romania). En: Dinosaur Eggs and Babies (Eds. K. Carpenter, K.F. Hirsch \& J.R. Horner). Cambridge University Press, Cambridge, 75-87.

Kohring, R. 1997. Eggshell structure as evidence in avian systematics. Preliminary results. Berliner Geowissenschaftliche Abhandlungen, E 25, 281-291.

López-Martínez, N., Moratalla, J.J. \& Sanz, J.L. 2000. Dinosaur nesting on tidal flats. Palaeogeography, Palaeoclimatology, Palaeoecology, 160, 153-163.

Masriera, A. \& Ullastre, J. 1983. Essai de synthèse stratigraphique des couches continentales de la fin du Crétacé des Pyrénées Catalanes (NE de l'Espagne). Geologie Mediterranéenne, 10, 283-290.

Mateus, I., Mateus, H., Telles, M., Mateus, O., Taquet, P., Ribeiro, V. \& Manupella, G. 1997. Couvée, oeufs et embryons d'un Dinosaure Théropode du Jurassique supérieur de Lourinhâ (Portugal). Comptes Rendus de l'Academie des Sciences Paris, Sciences de la terre et des planétes/ Earth \& Planetary Sciences, 325, 71-78.

Médus, J., Feist, M., Rocchia, R., Batten, D.J., Boclet, D., Colombo, F., Tambareau, Y. \& Villatte, J. 1988. Prospects 
for recognition of palynological Cretaceous/Tertiary boundary and an iridium anomaly in nonmarine facies of the eastern Spanish Pyrenees: a preliminary report. Newsletters Stratigraphy, 18, 123-138.

Mikhailov, K. E. 1997. Fossil and Recent Eggshell in Amniotic Vertebrates: fine Structure, comparative Morphology and Classification. Special Papers in Palaeontology, 56, $1-80$.

Moratalla, J.J. 1993. Restos indirectos de dinosaurios del registro español: Paleoicnología de la Cuenca de Cameros (Jurásico superior - Cretácico inferior) y Paleoología del Cretácico superior. Vol. II. Tesis doctoral. Universidad Autónoma de Madrid, 726 pp. (inédita).

Oms, O., Vicens, E. \& Galobart, À. 2002. Geological setting an dinosaur fossils of the Vallcebre syncline (North of the Barcelona province, Pyrenees, Spain). I Congreso Internacional sobre Dinosaurios y otros Reptiles Mesozoicos en España. Resúmenes.

Peitz, C. 2000. Megaloolithid dinosaur eggs from the Maastrichtian of Catalunya (NE-Spain)- Parataxonomic implications and stratigraphic utility. En: First International Symposium on Dinosaur Eggs and Babies, Isona. Extended Abstracts, 55-164.

Rosell, J., Linares, R. \& Llompart, C. 2001. El "Garumniense" prepirenaico. Revista de la Sociedad Geológica de España, 14, 47-56.
Sahni, A., Tandon, S.K., Jolly, A., Bajpai, S., Sood, A. \& Srinivasan, S. 1994. Upper Cretaceous dinosaur eggs and nesting sites from the Deccan volcano-sedimentary province of peninsular India. En: Dinosaur Eggs and Babies (Eds. K. Carpenter, K.F. Hirsch \& J. R. Horner). Cambridge University Press, Cambridge, 204-226.

Sanz, J.L., Moratalla, J.J., Díaz-Molina, M., López-Martínez, N., Kälin, O. \& Vianey-Liaud, M. 1995. Dinosaur nests at the sea shore. Nature, 376, 731-732.

Vianey-Liaud, M. \& López-Martínez, N. 1997. Late Cretaceous dinosaur eggshells from the Tremp Basin, southern Pyrenees, Lleida, Spain. Journal of Paleontology, 71, 1157-1171.

Vianey-Liaud, M., Mallan, P., Buscail, O. \& Montgelard, G. 1994. Review of French dinosaur eggshells: morphology, structure, mineral and organic composition. En: Dinosaur Eggs and Babies (Eds. K. Carpenter, K. F. Hirsch \& J.R. Horner). Cambridge University Press, Cambridge, 151-183.

Zhao Z. 1979. The advancement of researches on the dinosaurian eggs in China. In: Mesozoic and Cenozoic Red Beds of South China, Science Publishing Co., Beijing , 330-340.

Manuscrito recibido: 14 de Enero, 2004 Manuscrito aceptado: 15 de Abril, 2005 